\title{
Retrospectively acquired cohort study to evaluate the long-term impact of two different treatment strategies on disability outcomes in patients with relapsing multiple sclerosis (RE.LO.DI.MS): data from the Italian MS Register
}

\author{
Damiano Paolicelli ${ }^{1}$. . Giuseppe Lucisano ${ }^{2}$ - Alessia Manni ${ }^{1}$. Carlo Avolio ${ }^{3}$. Simona Bonavita ${ }^{4}$. \\ Vincenzo Brescia Morra ${ }^{5}$. Marco Capobianco ${ }^{6}$. Eleonora Cocco $^{7}$. Antonella Conte ${ }^{8,9}$. Giovanna De Luca ${ }^{10}$. \\ Francesca De Robertis ${ }^{11}$. Claudio Gasperini ${ }^{12}$. Maurizia Gatto ${ }^{13}$. Paola Gazzola ${ }^{14}$. Giacomo Lus ${ }^{4}$. \\ Antonio laffaldano $^{1}$. Pietro laffaldano ${ }^{1}$. Davide Maimone ${ }^{15}$. Giulia Mallucci ${ }^{16}$. Giorgia T. Maniscalco ${ }^{17}$. \\ Girolama A. Marfia ${ }^{18}$. Francesco Patti ${ }^{19}$. Ilaria Pesci ${ }^{20}$. Carlo Pozzilli ${ }^{21,22} \cdot$ Marco Rovaris $^{23}$. Giuseppe Salemi ${ }^{24}$. \\ Marco Salvetti ${ }^{25,26}$. Daniele Spitaleri ${ }^{27} \cdot$ Rocco Totaro $^{28} \cdot$ Mauro Zaffaroni $^{29}$. Giancarlo Comi ${ }^{30}$. \\ Maria Pia Amato ${ }^{31,32}$ - Maria Trojano on behalf of the Italian MS Register
}

Received: 11 July 2019 / Revised: 5 September 2019 / Accepted: 6 September 2019 / Published online: 18 September 2019

(c) Springer-Verlag GmbH Germany, part of Springer Nature 2019

\begin{abstract}
Background The increase in disease-modifying drugs (DMDs) allows individualization of treatment in relapsing multiple sclerosis (RMS); however, the long-term impact of different treatment sequences is not well established. This is particularly relevant for MS patients who may need to postpone more aggressive DMD strategies.

Objective To evaluate different therapeutic strategies and their long-term outcomes, measured as relapses and confirmed disability progression (CDP), in MS 'real-world' settings.

Methods Multicentre, observational, retrospectively acquired cohort study evaluating the long-term impact of different treatment strategies on disability outcomes in patients with RMS in the Italian MS Register.

Results We evaluated 1152 RMS-naïve patients after propensity-score adjustment. Patients included were receiving: interferon beta-1a (IFN- $\beta 1$ a) $44 \mu \mathrm{g}$ switching to fingolimod (FTY; IFN-switchers; $n=97$ ); FTY only (FTY-stayers; $n=157$ ); IFN- $\beta 1$ a only (IFN-stayers; $n=849)$. CDP and relapses did not differ between FTY-stayers and IFN-switchers [HR $(95 \%$ CI) $0.99(0.48-2.04), p=0.98$ and $0.81(0.42-1.58), p=0.55$, respectively]. However, IFN-stayers showed increased risk of relapses compared with FTY-stayers [HR (95\% CI) $1.46(1.00-2.12), p=0.05]$.

Conclusion The ideal treatment option for MS is becoming increasingly complex, with the need to balance benefit and risks. Our results suggest that starting with FTY affects the long-term disease outcome similarly to escalating from IFN- $\beta 1$ a to FTY.
\end{abstract}

Keywords Multiple sclerosis · Interferons · Long-term outcomes · Real-world setting · Treatment sequences · EDSS score

\section{Introduction}

Multiple sclerosis (MS) is a chronic, inflammatory, degenerative disorder of the brain and spinal cord with a prevalence of about 2.3 million individuals. Although the typical onset is in young adulthood, $3-5 \%$ of cases occur in childhood or adolescence. Approximately $80 \%$ of patients with MS follow a relapsing-remitting course of the disease, characterized by acute attacks and worsening of symptoms, followed by periods of remission $[1,2]$.
Damiano Paolicelli

damiano.paolicelli@uniba.it

Extended author information available on the last page of the article 
Therapeutic strategies in the MS landscape are rapidly changing. Disease-modifying drugs (DMDs), such as interferons (IFNs), have been widely used as first-line therapy in patients with relapsing MS (RMS). However, for many patients these injectable treatments have a suboptimal tolerability profile; moreover, they are only partially effective, and patients with RMS may need to switch from one DMD to another [3]. In particular, two different therapeutic approaches can be adapted to patients with RMS. Escalation consists of starting a first-line DMD early and if this is ineffective, switching to second-line drugs; whereas induction therapy supports the early use of second-line DMDs that could present safety concerns and are, therefore, usually reserved for non-responders.

In this context, there is an urgent need to bridge the knowledge gap between the availability of an increasing number of effective drugs and understanding how to offer the appropriate treatment to the right patient at the right time, gathering real-world data on long-term outcomes. As a consequence, understanding the long-term impact of different drugs is critical to define treatment sequences, as it still remains an issue to unravel [4].

Established evidence about a positive effect on the probability of reducing long-term disability progression is available, especially for injecting first-line therapies [5, 6]. Furthermore, a recent publication of a meta-analysis conducted to assess the first- and second-line therapies in patients with relapsing-remitting MS showed that IFN beta-1a (IFN- $\beta 1$ a) given subcutaneously (SC) three times weekly seemed to have the most favourable benefit-risk ratio among first-line DMDs [7].

In contrast, the TRANSFORMS clinical study showed the superiority of oral fingolimod (FTY) compared with intramuscular IFN- $\beta 1 \mathrm{a}$ in clinical and magnetic resonance imaging (MRI) parameters in patients with relapsing-remitting MS [8], despite data on efficacy of the oral drugs, including FTY, in preventing disability progression, not being conclusive, as indicated by results from the two pivotal phase 3 studies [8, 9]. In addition, the registration trial of FTY (FREEDOMS) [9] included a placebo group, and its longterm extension [10] showed that patients initially on placebo performed worse long term compared with FTY, even though they showed significant improvements in clinical and MRI measures, essentially replicating, in this within-group comparison, the findings from the between-group comparison in FREEDOMS. However, nowadays, the long-term efficacy profile of DMDs is demonstrated in head-to-head trials with a comparator group with active treatment. An approach that better resembles clinical practice and is more similar to the experience provided from the long-term extension of the TRANSFORMS study, in which the continuousFTY group showed a better control of annualized relapse rate compared with the IFN- $\beta 1$ a $30 \mu$ g-switch group, but disability progression and MRI outcomes at the end of the extension phase were not significantly different between the groups. This may have shown the possibility that different treatment approaches may have similar long-term effects on disability progression, providing a first input to the possibility to postpone FTY use during treatment sequencing [11].

The aim of our study was to evaluate long-term outcomes of different sequences of treatment, in terms of disability progression in RMS-naïve patients, by focusing on the direct comparison between two drugs, IFN- $\beta 1$ a $44 \mu \mathrm{g}$ and FTY, which both have a pivotal role in MS therapy, IFN- $\beta 1$ a being a standard first-line treatment, with consistent efficacy and safety data, and FTY being the first oral therapy.

Considering that big data registries offer the opportunity to study real-world clinical outcomes in large cohorts of patients, the strengths of our work are the 'real-world' setting, representative of daily clinical MS practice, and the huge cohort of patients collected in the Italian MS Register, the largest national clinical database, with about 140 Italian MS centres connected [12].

\section{Patients and methods}

\section{Study design}

We conducted a multicentre, observational, retrospectively acquired cohort study, to evaluate the long-term impact of different treatment strategies on disability outcomes in patients with RMS. Anonymized clinical records of patients with RMS, from their first treatment with IFN- $\beta 1$ a SC $44 \mu \mathrm{g}$ or FTY to their last follow-up on treatment with FTY or IFN- $\beta 1$ a SC $44 \mu \mathrm{g}$, were extracted from the Italian MS Register [12], between 1 January 2010 and May 2017. The decision on how and when to prescribe IFN or FTY was the responsibility of the treating neurologist at each MS centre. The time interval between IFN- $\beta 1$ a SC $44 \mu \mathrm{g}$ cessation and switching to FTY was considered as an untreated wash-out period. Main exclusion criteria were: patients who did not have a diagnosis of MS based on McDonald criteria 2010 [13] or progressive MS; patients who started with a different DMD to IFN- $\beta 1$ a SC $44 \mu \mathrm{g}$ or FTY or were exposed to a different DMD; patients with a wash-out period $\geq 3$ months after IFN- $\beta 1$ a $44 \mu \mathrm{g}$ cessation.

RMS-naïve patients meeting the eligibility criteria were divided into three groups: patients treated only with FTY until their final follow-up (FTY-stayers); patients who started with IFN- $\beta 1$ a SC $44 \mu \mathrm{g}$ and were switched to FTY, as first option, and received FTY until the final follow-up (IFN-switchers); patients receiving IFN- $\beta 1$ a SC $44 \mu \mathrm{g}$ from their first therapy until the final follow-up (IFN-stayers).

We evaluated two distinct comparisons. The first comparison was between FTY-stayers and IFN-switchers; in 
both cohorts, baseline was the first prescription of FTY. The second comparison was between IFN-stayers and FTY-stayers, where baseline was the first prescription of IFN or FTY, respectively. The switch from IFN to FTY was assessed on the basis of clinical activity. The outcome of the study was the evaluation of confirmed disability progression (CDP) and relapses from baseline to first event (for patients with an event) or last information at follow-up (for patients without an event).

CDP events were defined as $\geq 3$-month confirmed increases of: $\geq 0.5$ points for patients with a baseline Expanded Disability Status Scale (EDSS) score $>5.5$; $\geq 1.0$ point for those with a baseline EDSS score between 1 and 5.5, inclusive; and $\geq 1.5$ points for those with a baseline EDSS score of 0. A minimum of three visits (including baseline) with EDSS score evaluation was required. EDSS scores recorded within 30 days after the onset of a relapse were excluded.

\section{Statistical analysis}

The characteristics were expressed as median and interquartile range (IQR) or frequency and percentage for continuous and categorical covariates, respectively. The comparisons between groups (FTY-stayers vs IFN switchers and FTY stayers vs IFN stayers) were assessed with the $T$ test (or Mann-Whitney $U$ test as appropriate) for continuous variables and the $\chi^{2}$ statistic for categorical variables.

Cumulative incidence probability was reported according to the Kaplan-Meier method as explorative analysis. To minimize the imbalance, initial treatment effects were also adjusted by a propensity score (PS) in quintiles for age at baseline and EDSS score at baseline, relapses in the previous year, sex and clinical onset (monofocal vs multifocal), and disease duration from the onset to baseline. The evaluation of balance also considered squared transformation of continuous covariates and interaction terms between covariates. Multivariate Cox proportional hazard regression models adjusted for PS were performed to identify predictive factors for time to first relapse or first confirmed EDSS worsening event. Therefore, an algorithm generates a predicted probability for each subject (PS). The Cox models adjusted for PS were censored at 3 years to also balance for different length of follow-up.

To better underline differences in the two treatment strategies in mild-to-moderate patients, subgroup analyses were performed, stratifying patients for baseline EDSS score $(\leq 2$ and $>2)$, one previous relapse before baseline, and difference between first and baseline EDSS scores. $p$ values were two-sided, and $p<0.05$ was considered to be statistically significant. Analyses were carried out using R.

\section{Results}

Of 2319 patients selected from the Italian Register, 1167 were not eligible (Fig. 1).

Of the remaining 1152 patients (166 FTY-stayers, 112 IFN-switchers, and 874 IFN-stayers), a further 49 were excluded from the analysis because they had $<3$ visits. Therefore, data for 157 FTY-stayers, 97 IFNswitchers, and 849 IFN-stayers were analysed; however, the core analyses were in the two groups of FTY stayers and IFN switchers. The median time to IFN-switch was 54.5 months (IQR 38.9-90.1).

Baseline characteristics of the cohort by treatment group are shown in Table 1. The demographic and disease characteristics were generally similar between the FTY stayers and IFN switchers. In particular, most patients were female, with a monofocal onset, and a mild-to-moderate baseline EDSS score. In contrast, FTY stayers were significantly different from IFN stayers, with a higher age and EDSS score at baseline.

The descriptive results from survival analyses by Kaplan-Meier curves comparing FTY stayers with IFNswitchers are shown in Fig. 2. No differences in terms of Log-rank test were seen for CDP $(p=0.28)$ (HR 0.71; CI $0.38-1.32$ ) or relapses $(p=0.55)$ (HR 0.85; CI 0.49-1.47) during the follow-up in the two groups.

To simulate a randomization with respect to the treatment groups (IFN- $\beta 1 \mathrm{a} 44 \mu \mathrm{g}$ SC or FTY) and to cancel the imbalance with respect to the other characteristics associated with the outcome, we performed a PS adjustment. An algorithm generated a predicted probability for each subject (PS). After the PS adjustment, we did not observe any differences between FTY-stayers and IFN-switchers in CDP [hazard ratio (HR) $0.99 ; 95 \%$ confidence interval (CI) $0.48-2.04 ; p=0.98$ ] (Fig. 3).

Considering the effect on relapses, the two different treatment strategies showed overlapping results. We did not find statistically significant differences between the number of patients with relapses in either the FTY stayers or the IFN-switchers group (HR 0.81; 95\% CI 0.42-1.58; $p=0.55$ ).

The descriptive results from survival analyses by Kaplan-Meier curves comparing IFN stayers with FTY stayers are shown in Fig. 4. No differences in terms of Log-rank test were seen for CDP $(p=0.06)$ (HR 0.65; CI $0.41-1.01)$ or relapses $(p=0.08)(\mathrm{HR}=1.38$; CI 0.96-1.98) during the follow-up in the two groups. However, when we compared IFN stayers with FTY stayers, we found a higher risk of relapses in the IFN stayers group (HR 1.46; 95\% CI 1.00-2.12; $p=0.05$ ) (Fig. 4).

We also performed subgroup analyses, stratifying both FTY stayers and IFN switchers for baseline EDSS score 


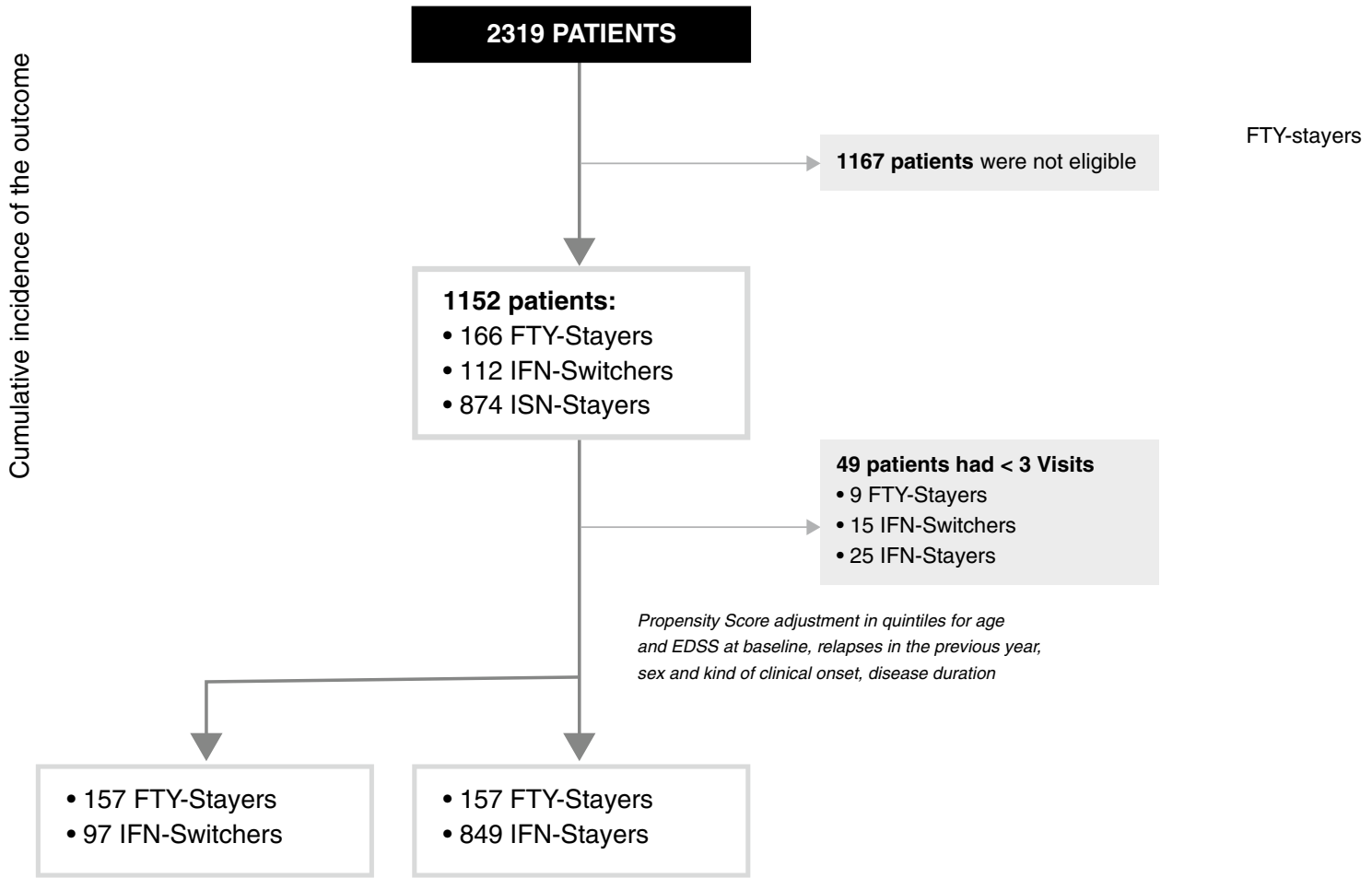

Fig. 1 Flow of the study. EDSS Expanded Disability Status Scale, FTY fingolimod, IFN interferon

Table 1 Demographic data at baseline

\begin{tabular}{|c|c|c|c|c|c|}
\hline & FTY-stayers $(n=157)$ & IFN-switchers $(n=97)$ & IFN-stayers $(n=849)$ & $\begin{array}{l}p \text { value FTY- } \\
\text { stayers vs IFN- } \\
\text { switchers }\end{array}$ & $\begin{array}{l}p \text { value FTY-stay- } \\
\text { ers vs IFN-stayers }\end{array}$ \\
\hline $\begin{array}{l}\text { Baseline age, years, median } \\
\text { (IQR) }\end{array}$ & $37.50(28.80-46.60)$ & $37.60(31.20-44.60)$ & $33.80(27.40-41.00)$ & 0.9629 & 0.0003 \\
\hline Baseline EDSS, median (IQR) & $2.50(1.50-3.50)$ & $3.00(2.00-4.00)$ & $2.00(1.00-2.50)$ & 0.0303 & $<0.0001$ \\
\hline Monofocal onset no. (\%) & $142(90.45)$ & $86(88.66)$ & $752(88.57)$ & 0.6482 & 0.4935 \\
\hline Females no $(\%)$ & $94(59.87)$ & $67(69.07)$ & $577(67.96)$ & 0.1392 & 0.0482 \\
\hline $\begin{array}{l}\text { No. of relapses, } 2 \text { years before, } \\
\text { median (IQR) }\end{array}$ & $2(1-2)$ & $2(1-3)$ & $2(1-2)$ & 0.3607 & 0.434 \\
\hline No. of visits, median (IQR) & $7(5-11)$ & $8(6-12)$ & $8(4-13)$ & 0.111 & $<0.0001$ \\
\hline $\begin{array}{l}\text { Length of follow-up, months, } \\
\text { median (IQR) }\end{array}$ & $39.1(27.7-54.5)$ & $32.7(16.3-46.5)$ & $62.3(39.2-99.5)$ & 0.001 & $<0.0001$ \\
\hline $\begin{array}{l}\text { Disease duration, months, } \\
\text { median (IQR) }\end{array}$ & $37.1(13.4-107.8)$ & $102.9(66.7-154.4)$ & $25.2(9.0-84.1)$ & $<0.0001$ & 0.03 \\
\hline $\begin{array}{l}\text { At least a relapse on follow-up, } \\
\text { no. }(\%)\end{array}$ & $33(21.02)$ & $21(21.65)$ & $251(29.56)$ & 0.91 & 0.03 \\
\hline CDP, no. $(\%)$ & $24(15.29)$ & $17(17.53)$ & $93(10.95)$ & 0.64 & 0.12 \\
\hline Time to relapse, median (IQR) & $2.80(1.63-3.87)$ & $2.09(1.08-3.33)$ & $3.31(1.79-5.73)$ & 0.0073 & 0.0005 \\
\hline Time to CDP, median (IQR) & $2.91(2.07-4.04)$ & $2.03(1.08-3.69)$ & $4.25(2.68-6.98)$ & 0.0005 & $<0.0001$ \\
\hline
\end{tabular}

$C D P$ confirmed disability progression, EDSS Expanded Disability Status Scale, FTY fingolimod, IFN interferon, IQR interquartile range

$(\leq 2$ or $>2)$ and the number of relapses during the year pre-baseline ( 1 or $>1)$. In patients with an EDSS score $\leq 2$, no statistically significant differences were found at 3-month CDP between the two groups $(p=0.56)$; the same result was obtained in patients with an EDSS score $>2(p=0.64)$. Considering the number of relapses, 
Fig. 2 Kaplan-Meier curves comparing fingolimod (FTY)-stayers and interferon (IFN)-switchers according to confirmed disability progression (CDP) at 3 months of follow-up (a) and relapses (b)

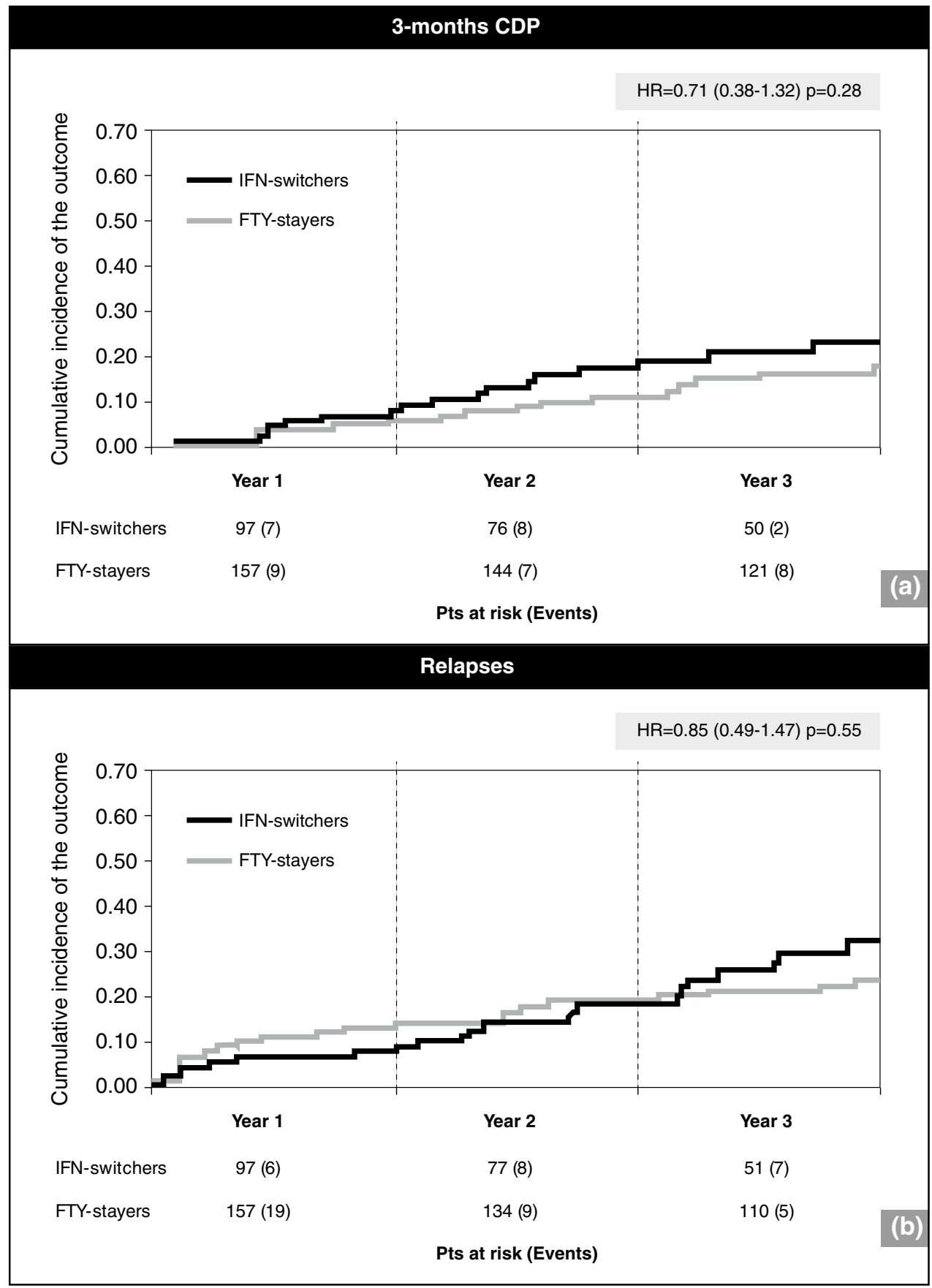

patients with only one relapse in the year pre-baseline did not show a different risk of CDP $(p=0.79)$ than patients with an annualized change in EDSS score $(\Delta \mathrm{EDSS}) \geq 1$ and more than one previous relapse $(p=0.99)$ (Table 2$)$.

No statistically significant differences were found between the two groups in the relapse rate in patients with a baseline EDSS score $\leq 2$ (HR 0.70; 95\% CI 0.28-1.77; $p=0.46)$, patients with a baseline EDSS score $>2$ (HR $0.84 ; 95 \%$ CI $0.39-1.82 ; p=0.79)$, patients with one previous relapse (HR $0.49 ; 95 \%$ CI $0.21-2.13 ; p=0.67$ ), and patients with $\Delta \mathrm{EDSS} \geq 1$ and more than one previous relapse (HR 4.46; 95\% CI 0.65-30.37; $p=0.13$ ) (Table 2).

\section{Discussion}

The introduction of DMDs for RMS has led to a reduction in disease activity associated with a demonstrable improvement in clinical and radiological outcomes. Nevertheless, owing to the chronic nature of the disease, patients with 
Fig. 3 Analysis of treatment effects in confirmed disability progression (CDP) and relapse. a Fingolimod (FTY)-stayers were compared with interferon (IFN)-switchers; b FTY-stayers were compared with IFN-stayers. (Asterisk) The treatment effects were explored by a propensity-score adjustment in quintiles for age, duration of disease from onset, Expanded Disability Status Scale at baseline, relapses in the previous year, sex, and clinical onset. $C I$ confidence interval, $H R$ hazard ratio

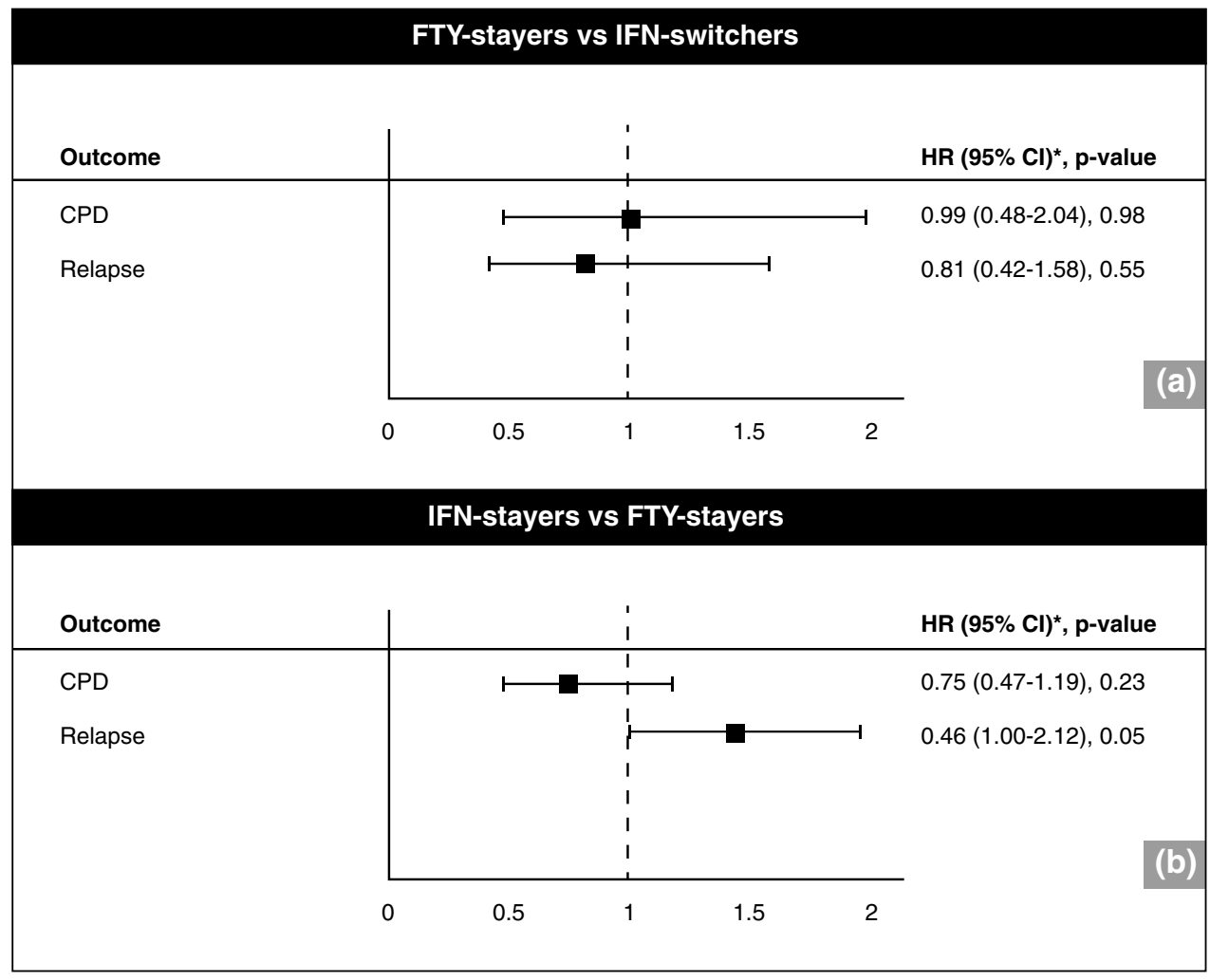

MS may require a change in therapy over time because of suboptimal response, safety concerns, intolerable side effects to the DMD, or a change in the risk tolerance of the patient [14]. In this setting, the introduction of FTY, as the first oral therapy, has strongly improved patient compliance, broadening therapeutic options with a high-efficacy DMD. However, it is the neurologist's choice whether more aggressive DMDs should be used as first treatment, and in which cases. The 2018 guidelines for MS treatment from the European Committee for Treatment and Research in Multiple Sclerosis and the American Academy of Neurology underline the importance of early treatment of the disease $[15,16]$. At the same time, sequencing of DMDs for each patient should be designed to better control disease activity, while minimizing the risk of disability or relapse. Previous results showed the superior efficacy of oral FTY with respect to relapse rates in MS patients compared with intramuscular IFN- $\beta 1$ a [8]. This result seemed to support an early initiation of high-efficacy therapy, also considering that every DMD has a greater efficacy at earlier stages of the disease course, that the risk of serious adverse events (opportunistic infections/malignancies) is lower at younger ages, and that therapeutic options to reverse disability are not available $[17,18]$. However, the choice of the best DMD depends on an accurate balance of the benefit-risk ratio, after accounting for specific diseaseand patient-related factors, at a specific point in time. An escalation paradigm, in which safer first-line therapies are used at the beginning of MS, must be considered in such a chronic disease, because early high-efficacy DMDs may expose patients to risks that increase with longer use and the relatively recent introduction of these newest DMDs, brings unknown long-term safety concerns.

Nowadays, patients with suboptimal responders can be identified with specific scores $[19,20]$ in a reasonably short time, before causing substantial worsening of disability. The growing importance of sequencing strategies in the treatment of MS led to the design of a wide randomized controlled trial, TREAT-MS (ClinicalTrials.gov Identifier: NCT03500328) which is currently ongoing and is aiming to evaluate whether an 'early aggressive' therapy approach, versus starting with a traditional, first-line therapy, influences the intermediate-term risk of disability.

Our study also aimed to address the sequencing issue, evaluating the long-term impact of the different treatment strategies on CDP and the risk of relapses.

According to our results, IFN- $\beta 1 \mathrm{a} 44 \mu \mathrm{g}$ and FTY appear to be equal in terms of effectiveness on disability progression in MS. This outcome is particularly valuable in the population with mild-to-moderate MS, as this allows the clinician to personalize and tailor the therapy to the patient's needs and to balance the benefit-risk ratio.

To achieve the prevention of relapses and disability accumulation, the neurologist needs to carefully plan the sequencing of treatments. This means estimating treatment 
Fig. 4 Kaplan-Meier curves comparing fingolimod (FTY) stayers and interferon (IFN) stayers according to relapses (a) and confirmed disability progression (CDP) at 3 months of follow-up (b)

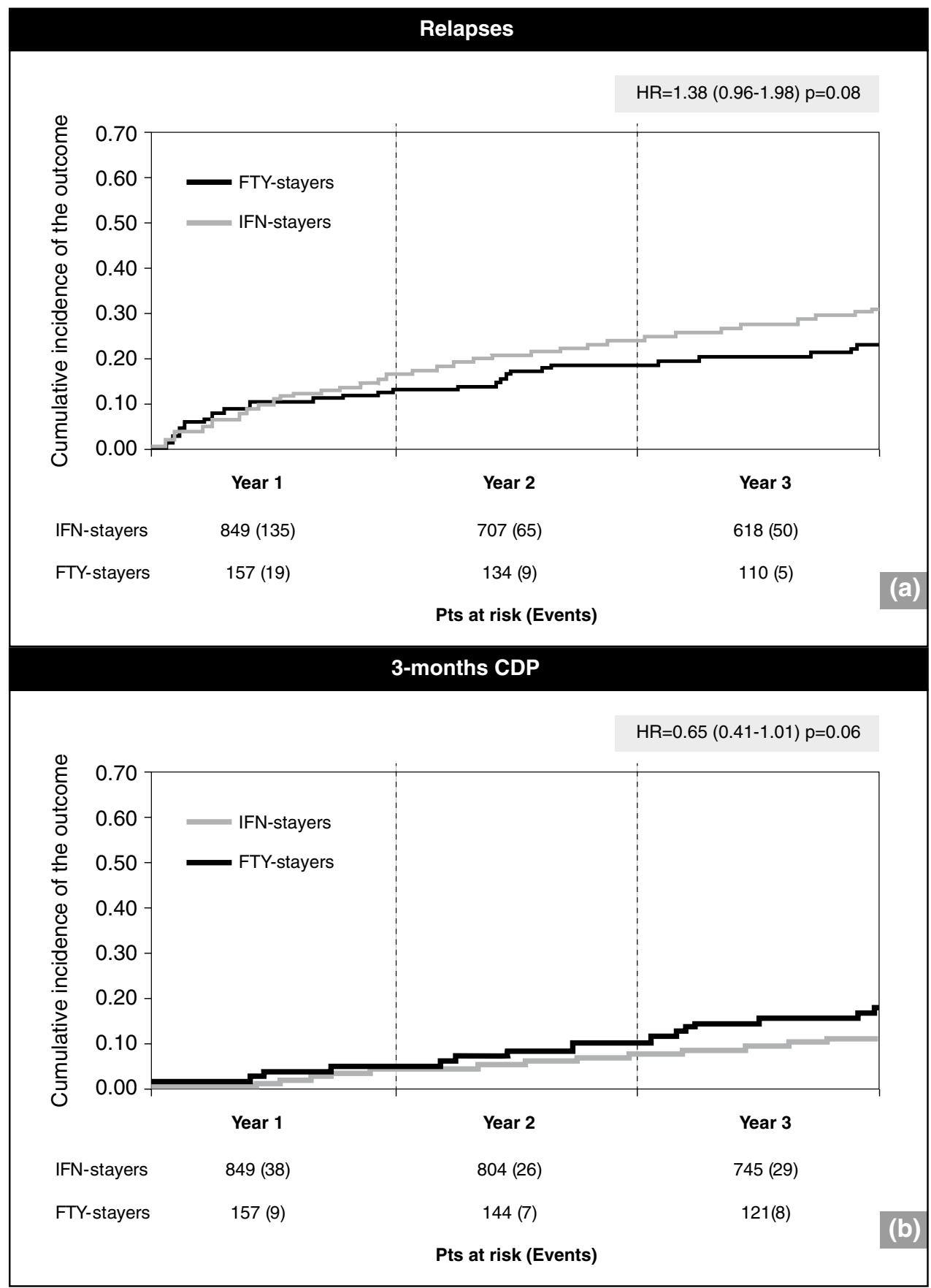

efficacy and safety, along with balancing individual patient preferences, adherence, and characteristics. Our study specifically addresses these issues; whereas drugs trials, which are head-to-head comparisons, do not represent this realworld MS setting. Therefore, our data represent an advancement in understanding the effectiveness of treatments in realworld patients.

In November 2018 (after the completion of our study), FTY obtained regulatory approval in Europe for children and adolescents, 10-17 years of age, with relapsing-remitting MS. However, the long-term outcomes of DMDs on the immune system, and therefore their possible role in the onset of oncological diseases, are not yet fully understood. For this reason, it would be better to use more aggressive treatments with caution in young people. In addition, in Europe FTY is considered a second-line DMD after failure of a first-line agent for patients with highly active disease [14]. We know that an even longer period of follow-up should be required to confirm our results; however, currently a real-world followup of more than 4 years is not available with the new treatments, and our study was pioneering when we planned its design. 
Table 2 Subgroup analysis of treatment effects in terms of $\mathrm{CDP}$ and of relapse

\begin{tabular}{lllll}
\hline & $\begin{array}{l}\text { No. of } \\
\text { FTY- } \\
\text { stayers }\end{array}$ & $\begin{array}{l}\text { No. of IFN- HR (95\% CI) } \\
\text { switchers }\end{array}$ & $p$ value \\
\hline
\end{tabular}

\section{CDP}

$\begin{array}{llll}\text { Baseline EDSS score } \leq 2 & 78 & 37 & 0.73(0.37-1.37) 0.56 \\ \text { Baseline EDSS score }>2 & 79 & 60 & 0.83(0.37-1.86) 0.64 \\ \text { One relapse before baseline } & 51 & 29 & 0.86(0.29-2.55) 0.79 \\ \Delta \text { EDSS } \geq 1 \text { and }>1 \text { relapse before baseline } & 14 & 37 & 0.00(0.00-1.00) 0.99 \\ \text { At least a relapse during follow-up } & & & \\ \text { Baseline EDSS score } \leq 2 & 78 & 37 & 0.70(0.28-1.77) 0.46 \\ \text { Baseline EDSS score }>2 & 79 & 60 & 0.84(0.39-1.82) 0.79 \\ \text { One relapse before baseline } & 51 & 29 & 0.49(0.21-2.13) 0.67 \\ \Delta \text { EDSS } \geq 1 \text { AND }>1 \text { relapse before baseline } & 14 & 37 & 4.46(0.65-30.37) 0.13\end{array}$

$C D P$ confirmed disability progression, $\triangle E D S S$ annualized change in EDSS score, $C I$ confidence index, EDSS Expanded Disability Status Scale, $F T Y$ fingolimod, $H R$ hazard ratio, $I F N$ interferon
Considering the growing importance of the sustainability of drug treatments for the health system, the demonstration of cost-effectiveness becomes another crucial point to consider in best clinical practice. FTY therapy requires a higher drug-acquisition cost compared with IFN- $\beta 1$ a [21]; therefore, choice of the latter would result in substantial savings for the national health service. Unlike previous studies [8], the RELODIMS study did not evaluate MRI data. This could be a limitation, as the current criteria for defining the efficacy of a treatment in MS, also called NEDA (No Evidence of Disease Activity), is a composite outcome that has to take into account the presence of new or active (enhancing) lesions on MRI scans. This result is missing in many real-world studies.

In conclusion, we demonstrated the feasibility of a sequencing approach in mild-to-moderate patients, initiated with IFN- $\beta 1$ la early in the disease course and then switched to FTY. This strategy may represent an advantage in the management of selected patients. In our real-world study, we evaluated a comparator group with active treatments and the results clearly show that sequencing from a safer drug to FTY similarly affects the long-term disease outcome as an approach that starts using FTY like a more aggressive therapy.

\section{Compliance with ethical standards}

Conflicts of interest G. Lucisano, A. Manni, S. Bonavita, V. Brescia Morra, A. Conte, F. De Robertis, P. Gazzola, A. Iaffaldano, C. Gasperini, M. Gatto, G.T. Maniscalco, I. Pesci report no disclosures relevant to the manuscript. D. Paolicelli received advisory board membership, speakers honoraria, travel support, research grants, consulting fees, or clinical trial support from Almirall, Bayer Schering, Biogen, Celgene, Excemed, Genzyme, Merck, Mylan, Novartis, Sanofi, Roche, Teva. M.P. Amato has received research grants and honoraria as a speaker and member of advisory boards by Biogen, Merck, Sanofi Genzyme, Teva, Novartis, Roche. C. Avolio served on scientific advisory boards for Merck, Sanofi-Genzyme and Roche, and has received travel and/ or speaker honoraria from Merck, Roche, Teva, Biogen, Novartis and Sanofi-Genzyme. M. Capobianco received personal compensation for speaking at meeting or participating in advisory board from Almirall, Biogen, Merck, Novartis, Sanofi, Roche. E. Cocco has served on scientific Advisory Boards for Bayer, Biogen, Merck, Novartis, Roche, Sanofi Genzyme and TEVA; she also received speaker honoraria and research grants for her department by the same companies. G. Comi received personal compensation for consulting services and/or speaking activities from Novartis, Teva, Sanofi, Sanofi-Genzyme, Merck, Biogen, Excemed, Serono Symposia International Foundation, Roche, Almirall, Receptos, Celgene, and Forward Pharma. G. De Luca served on scientific advisory boards for Merck, Sanofi-Genzyme and Roche, and has received travel and/or speaker honoraria from Merck, Roche, Teva, Biogen, Novartis and Sanofi-Genzyme. G. Lus receveid travel fundings, research support, speaker honoraria from: Biogen, Novartis, Sanofi Genzyme, Bayer, Teva, Almirall, Allergan, Ipsen, Merck, Merz Pharma Italia. P. Iaffaldano received advisory board membership, speaker honoraria, travel support, research grants, consulting fees, or clinical trial support from Almirall, Bayer Schering, Biogen, Celgene, Excemed, Genzyme, Merck, Mylan, Novartis, Sanofi, Roche, Teva. D. Maimone receveid travel funds and speaker honoraria from Biogen, Novartis, Sanofi Genzyme, Teva, Almirall, Merck, Roche. G. Mallucci received support to travel to scientific meetings from Bayer Schering, Biogen, Genzyme, Merck Serono, Novartis, Roche, Sanofi-Aventis, Teva; received research grants for the MS Center at IRCCS Mondino, Pavia, Italy from Biogen and served on the scientific advisory board for Biogen, Genzyme and Merck Serono. G.A. Marfia is an Advisory Board member of Biogen Idec, Genzyme, Merck-Serono, Novartis, Teva and received honoraria for speaking or consultation fees from Almirall, Bayer Schering, Biogen Idec, Merck Serono, Novartis, Sanofi-Genzyme, Teva. She is the principal investigator in clinical trials for Actelion, Biogen Idec, Merck Serono, Mitsubishi, Novartis, Roche, Sanofi-Genzyme, Teva. F. Patti has received honoraria for speaking activities by Bayer Schering, Biogen, Merck, Novartis, Roche, TEVA and Sanofi Aventis; he also served as advisory board member the following companies: Bayer Schering, Roche, Biogen, Merck, Novartis; he was also funded by Pfizer and FISM for epidemiological studies; finally he received grant for congress participation from Bayer Schering, Roche, Biogen, Merck, Novartis, Sanofi Aventis, and TEVA. C. Pozzilli has received consultant fees from Actelion, Biogen, Genzyme, Merck-Serono, Novartis, Teva Neurosciences and grant or research support from Biogen, Merck-serono, Novartis and Teva neuroscinces. M. Rovaris received fees for consulting or scientific speaking from Almirall, Biogen, Genzyme-Sanofi, Merck Serono and TEVA. G. Sa- 
lemi received grants and honoraria by Almirall, Biogen-Dompè, Merck-Serono, Novartis, Roche, Sanofi-Aventis, and TEVA. M. Salvetti received research support and consulting fees from Biogen, Merck, Novartis, Roche, Sanofi, Teva. D. Spitaleri received fees for consulting or congress participation from Novartis, TEVA, Sanofi and Biogen. R. Totaro has served on advisory boards and/or received honoraria for speaking or consultation fees from Biogen, CSL behring, MerckSerono, Novartis, Roche, Sanofi-Genzyme, Shire, Teva. M. Zaffaroni received personal financial support for attending scientific meetings from Merck and financial support for his Department from Novartis. M. Trojano received advisory board membership, speaker honoraria, travel support, research grants, consulting fees, or clinical trial support from Actelion, Allergan, Almirall, Bayer Schering, Biogen, Celgene, Excemed, Genzyme, Merck, Mylan, Novartis, Sanofi, Roche, Teva.

Ethic approval The study was conducted in accordance with the ethical standards of the responsible committee on human experimentation and with the Helsinki Declaration of 1975 . The study was approved by the Ethic Committee of AOU Policlinico, Bari on 17 July 2018; ID number 0060669 .

\section{References}

1. Freedman MS, Selchen D, Prat A, Giacomini PS (2018) Managing multiple sclerosis: treatment initiation, modification, and sequencing. Can J Neurol Sci 45(5):489-503

2. Oh J, Vidal-Jordana A, Montalban X (2018) Multiple sclerosis: clinical aspects. Curr Opin Neurol 31(6):752-759

3. Gross RH, Corboy JR (2019) Monitoring, switching, and stopping multiple sclerosis disease-modifying therapies. Continuum (Minneap Minn) 25(3):715-735

4. Sormani MP, Bruzzi P (2015) Can we measure long-term treatment effects in multiple sclerosis? Nat Rev Neurol 11(3):176-182

5. Signori A, Gallo F, Bovis F et al (2016) Long-term impact of interferon or Glatiramer acetate in multiple sclerosis: a systematic review and meta-analysis. Mult Scler Relat Disord 6:57-63

6. Kappos L, Kuhle J, Multanen J et al (2015) Factors influencing long-term outcomes in relapsing-remitting multiple sclerosis: PRISMS-15. J Neurol Neurosurg Psychiatry 86:1202-1207

7. Mendes D, Alves C, Batel-Marques F (2016) Benefit-risk of therapies for relapsing-remitting multiple sclerosis: testing the number needed to treat to benefit (NNTB), number needed to treat to harm (NNTH) and the likelihood to be helped or harmed (LHH): a systematic review and meta-analysis. CNS Drugs 30:909-929
8. Cohen JA, Barkhof F, Comi G et al (2010) Oral fingolimod or intramuscular interferon for relapsing multiple sclerosis. N Engl J Med 362:402-415

9. Kappos L, Radue EW, O'Connor P et al (2010) A placebo-controlled trial of oral fingolimod in relapsing multiple sclerosis. $\mathrm{N}$ Engl J Med 362:387-401

10. Kappos L, O'Connor P, Radue EW et al (2015) Long-term effects of fingolimod in multiple sclerosis: the randomized FREEDOMS extension trial. Neurology 84:1582-1591

11. Cohen JA, Khatri B, Barkhof F et al (2016) Long-term (up to 4.5 years) treatment with fingolimod in multiple sclerosis: results from the extension of the randomised TRANSFORMS study. $\mathbf{J}$ Neurol Neurosurg Psychiatry 87:468-475

12. Trojano M, Bergamaschi R, Amato MP et al (2019) The Italian multiple sclerosis register. Neurol Sci 40:155-165

13. Polman CH, Reingold SC, Banwell B et al (2011) Diagnostic criteria for multiple sclerosis: 2010 revisions to the McDonald criteria. Ann Neurol 69:292-302

14. Pardo G, Jones DE (2017) The sequence of disease-modifying therapies in relapsing multiple sclerosis: safety and immunologic considerations. J Neurol 264:2351-2374

15. Montalban X, Gold R, Thompson AJ et al (2018) ECTRIMS/ EAN guideline on the pharmacological treatment of people with multiple sclerosis. Mult Scler 24:96-120

16. Rae-Grant A, Day GS, Marrie RA et al (2018) Practice guideline recommendations summary: disease- modifying therapies for adults with multiple sclerosis: report of the Guideline Development, Dissemination, and Implementation Subcommittee of the American Academy of Neurology. Neurology 90:777-788

17. Comi G, Radaelli M, Soelberg Sørensen P (2017) Evolving concepts in the treatment of relapsing multiple sclerosis. Lancet 389:1347-1356

18. Corboy JR, Weinshenker BG, Wingerchuk DM (2018) Comment on 2018 American Academy of Neurology guidelines on diseasemodifying therapies in MS. Neurology 90:1106-1112

19. Rio J, Castilló J, Rovira A et al (2009) Measures in the first year of therapy predict the response to interferon beta in MS. Mult Scler $15: 848-853$

20. Sormani MP, Rio J, Tintorè $M$ et al (2013) Scoring treatment response in patients with relapsing multiple sclerosis. Mult Scler 19:605-612

21. Lee S, Baxter DC, Limone B et al (2012) Cost-effectiveness of fingolimod versus interferon beta-1a for relapsing remitting multiple sclerosis in the United States. J Med Econ 15:1088-1096

\section{Affiliations}

Damiano Paolicelli ${ }^{1} \odot$. Giuseppe Lucisano ${ }^{2}$. Alessia Manni ${ }^{1} \cdot$ Carlo Avolio $^{3}$ - Simona Bonavita ${ }^{4}$ Vincenzo Brescia Morra ${ }^{5}$. Marco Capobianco ${ }^{6}$. Eleonora Cocco ${ }^{7}$. Antonella Conte ${ }^{8,9}$. Giovanna De Luca ${ }^{10}$. Francesca De Robertis ${ }^{11}$. Claudio Gasperini ${ }^{12}$. Maurizia Gatto ${ }^{13}$. Paola Gazzola ${ }^{14}$. Giacomo Lus ${ }^{4}$. Antonio laffaldano ${ }^{1}$. Pietro laffaldano ${ }^{1}$. Davide Maimone ${ }^{15}$. Giulia Mallucci ${ }^{16}$. Giorgia T. Maniscalco ${ }^{17}$. Girolama A. Marfia ${ }^{18}$. Francesco Patti ${ }^{19}$. Ilaria Pesci ${ }^{20}$. Carlo Pozzilli ${ }^{21,22}$. Marco Rovaris ${ }^{23}$. Giuseppe Salemi ${ }^{24}$. Marco Salvetti ${ }^{25,26}$. Daniele Spitaleri ${ }^{27} \cdot$ Rocco Totaro $^{28} \cdot$ Mauro Zaffaroni $^{29}$. Giancarlo Comi ${ }^{30}$. Maria Pia Amato ${ }^{31,32}$. Maria Trojano' on behalf of the Italian MS Register

1 Department of Basic Medical Sciences, Neurosciences, and Sense Organs, Multiple Sclerosis Center, University of Bari "Aldo Moro", Bari, Italy
2 Center for Outcomes Research and Clinical Epidemiology, Pescara, Italy

3 Department of Medical and Surgical Sciences, University of Foggia, Foggia, Italy 
4 Department of Advanced Medical and Surgical Sciences, University of Campania "Luigi Vanvitelli", Napoli, Italy

5 Department of Neurosciences, Reproductive and Odontostomatological Sciences, "Federico II" University of Naples, Naples, Italy

6 Department of Neurology and Regional Multiple Sclerosis Centre, University Hospital San Luigi, Orbassano, TO, Italy

7 Department of Medical Science and Public Health, University of Cagliari and Multiple Sclerosis Center, Cagliari, Italy

8 Department of Human Neurosciences, Sapienza, University of Rome, Rome, Italy

9 IRCCS Neuromed, Pozzilli, IS, Italy

10 Neurology Clinic, Multiple Sclerosis Centre, SS Annunziata Hospital, Chieti, Italy

11 Department of Neurology, Vito Fazzi Hospital, ASL, Lecce, Italy

12 Department of Neuroscience, San Camillo Hospital, Rome, Italy

13 Neurology Unit, "F. Miulli" Hospital, Acquaviva delle Fonti BA, Italy

14 Departemental Center for the Diagnosis and Treatment of Demyelinating Diseases, Sestri Ponente, Genoa, Italy

15 Multiple Sclerosis Center, Garibaldi Hospital, Catania, Italy

16 Multiple Sclerosis Center of IRCCS Mondino Foundation, Pavia, Italy

17 Multiple Sclerosis Regional Center, A. Cardarelli Hospital, Naples, Italy

18 Department of Systems Medicine, Multiple Sclerosis Clinical and Research Center, University of Rome Tor Vergata, Rome, Italy
19 Department "G.F. Ingrassia", Multiple Sclerosis Center, University of Catania, Catania, Italy

20 Multiple Sclerosis Center, Ospedale di Vaio (I.P.), Fidenza, PR, Italy

21 Multiple Sclerosis Center, Sant'Andrea Hospital, Sapienza University of Rome, Rome, Italy

22 Department of Neurology, University La Sapienza, Rome, Italy

23 Multiple Sclerosis Center, IRCCS Fondazione Don Carlo Gnocchi, Milan, Italy

24 Department of Biomedicine, Neuroscience and Advanced Diagnostics, Palermo University, Palermo, Italy

25 Department of Neuroscience, Mental Health and Sensory Organs, Sapienza University, Rome, Italy

26 Istituto Neurologico Mediterraneo (INM) Neuromed, Pozzilli, Isernia, Italy

27 UOC Neurology, AORN San G. Moscati, Avellino, Italy

28 Department of Neurology, Demyelinating Disease Center, San Salvatore Hospital, L'Aquila, Italy

29 Multiple Sclerosis Center, Hospital of Gallarate, Gallarate, Italy

30 Department of Neurology, San Raffaele Hospital, Milan, Italy

31 Department NEUROFARBA, University of Florence, Florence, Italy

32 IRCCS and Fondazione Don Carlo Gnocchi, Florence, Italy 